\title{
Numerical Approximation of Minimum Norm Solutions of $K f=g$ for Special $K$
}

\author{
By Glenn R. Luecke
}

\begin{abstract}
Let $K: L_{2}(Y, \mu) \rightarrow L_{2}(X, \nu)$ be continuous and linear and assume $(K f)(x)=$ $\int_{Y} k(x, y) f(y) d \mu(y)$. Define $k_{x}$ by $k_{x}(y)=k(x, y)$. Assume $K$ has the property that (a) $k_{x} \in L_{2}(Y, \mu)$ for all $x \in X$ and (b) if $K f=0 \nu$-a.e., then $(K f)(x)=0$ for all $x \in X$. For example, if $X=Y=[0,1], \mu=\nu$ is Lebesgue measure and if $k(x, y)$ satisfies a Lipschitz condition in $x$, then $K$ has the above property. Assume $K$ satisfies this property and $f_{0}$ is a minimum $L_{2}$ norm solution of the first-kind integral equation $(K f)(x)=g(x)$ for all $x \in X$. It is shown that $f_{0}$ is the $L_{2}$-norm limit of linear combinations of the $k_{x_{i}}$ 's. It is then shown how to choose constants $c_{1}, \ldots, c_{n}$ to minimize $\left\|f_{0}-\sum_{j=1}^{n} c_{j} k_{x_{j}}\right\|$ without knowing what $f_{0}$ is. This paper also contains results on how to choose the $k_{x_{j}}$ 's as well as numerical examples illustrating the theory.
\end{abstract}

Introduction. Let $K$ be a continuous linear transformation mapping $L_{2}(Y, \mu)$ into $L_{2}(X, \nu)$ defined by $(K f)(x)=\int_{Y} k(x, y) f(y) d \mu(y) ; k(x, y)$ is called the kernel of the integral operator $K$ and is always assumed to be in $L_{2}(X \times Y, \nu \times \mu)$. Define $k_{x}$ to be $k_{x}(y)=k(x, y)$. This paper considers the numerical approximation of the minimum norm solution of the first-kind integral equation $(K f)(x)=g(x)$ for all $x \in X$ for a special class of integral operators $K$. For background information concerning first-kind equations, see [1].

1. Theoretical Results. The following property of the integral operator $K$ is a key to the development of the theory.

Definition 1.1. The integral operator $K$ with kernel function $k(x, y)$ is said to satisfy property $(\mathrm{C})$ if

(a) $k_{x} \in L_{2}(Y, \mu)$ for all $x \in X$, and

(b) if $(K f)(x)=0 \nu$-a.e. for some $f \in L_{2}(Y, \mu)$, then $(K f)(x)=0$ for all $x \in X$.

Example 1.2. Let $X$ be an open connected set in Euclidean $n$-space and let $\nu$ be Lebesgue measure on $X$. Assume $\mu(Y)<\infty$. Assume $k_{x} \in L_{2}(Y, \mu)$ for all $x$ and assume $k(x, y)$ is continuous in $x \in X$ uniformly in $y \in Y$, i.e., for each $x_{0} \in X$ and $\varepsilon>0$ there exists $\delta>0$ such that if $x \in X$ such that $\left|x-x_{0}\right|<\delta$ then $\left|k(x, y)-k\left(x_{0}, y\right)\right|<\varepsilon$ for all $y \in Y$. Using the Schwarz inequality and routine real analysis, it follows that $K$ satisfies property (C). An example of such a $K$ is $(K f)(x)=\int_{0}^{1} k(x, y) f(y) d y, 0 \leq x \leq 1$, where the partial derivative of $k(x, y)$ with respect to $x$ is a continuous function of $x$ and $y$, or if $k(x, y)$ is a continuous function of $x$ and $y$ satisfying the Lipschitz condition, $\left|k\left(x_{1}, y\right)-k\left(x_{2}, y\right)\right| \leq M\left|x_{1}-x_{2}\right|^{\alpha}$

Received March 13, 1986; revised April 5, 1988.

1980 Mathematics Subject Classification (1985 Revision). Primary 65R20, 45B05,47A50.

Key words and phrases. First-kind integral equation, numerical solution of first-kind integral equations. 
for all $x_{1}, x_{2}$, and $y$, where $M$ and $\alpha$ are positive constants (independent of $x_{1}, x_{2}$, $X, y$ and $Y)$.

Let $N(K)$ denote the null space of $K$ and let $M^{\perp}$ denote the orthogonal complement of any set $M$, i.e., $M^{\perp}=\{f:(f, g)=0$ for all $g \in M\}$. Here $(f, g)$ denotes the inner product of $f$ and $g$. The goal is to find minimal hypotheses on the operator $K$ so that $N(K)^{\perp}$ is equal to the $L_{2}$-closed span of the $k_{x}^{\prime} s, x \in X$. For simplicity of exposition, it is assumed that all functions are real-valued.

THEOREM 1.3. If $k_{x} \in L_{2}(Y, \mu)$ for all $x \in X$, then

$$
N(K)^{\perp} \subset \text { closed } \operatorname{span}\left\{k_{x}: x \in X\right\} .
$$

Proof. Let $M=\left\{k_{x}: x \in X\right\}$ and let $f_{0} \in M^{\perp}$. Then for all $x \in X,\left(f_{0}, k_{x}\right)=0$. Thus, for $x \in X$,

$$
\left(K f_{0}\right)(x)=\int_{Y} k(x, y) f_{0}(y) d \mu(y)=\int_{Y} k_{x}(y) f_{0}(y) \mu(y)=\left(k_{x}, f_{0}\right)=0 .
$$

The second to last equality above is valid because it is assumed that all functions are real-valued. It follows that $f_{0} \in N(K)$. Therefore, $M^{\perp} \subset N(K)$. Then it follows that $N(K)^{\perp} \subset M^{\perp \perp}$. But $M^{\perp \perp}$ is the closed span of $M$ and the proof is complete.

THEOREM 1.4. If operator $K$ satisfies property $(\mathrm{C})$, then

$$
N(K)^{\perp}=\text { closed } \operatorname{span}\left\{k_{x}: x \in X\right\} .
$$

Proof. Let $M=$ closed $\operatorname{span}\left\{k_{x}: x \in X\right\}$ and let $f_{0} \in N(K)$. Then $\left(K f_{0}\right)(x)=0$ $\nu$-a.e. Since $K$ satisfies property $(\mathrm{C}),\left(K f_{0}\right)(x)=0$ for all $x \in X$. Then for $x \in X$,

$$
\left(f_{0}, k_{x}\right)=\int_{Y} k_{x}(y) f_{0}(y) d \mu(y)=\int_{Y} k(x, y) f_{0}(y) d \mu(y)=\left(K f_{0}\right)(x)=0 .
$$

It follows that $k_{x} \in N(K)^{\perp}$ for all $x \in X$. Thus, $M \subset N(K)^{\perp}$. By Theorem 1.3, $N(K)^{\perp} \subset M$ and hence $M=N(K)^{\perp}$ and the theorem is proved.

Recall $f_{0}$ is a minimum norm solution of $(K f)(x)=f(x)$ if and only if $\left(K f_{0}\right)(x)=$ $g(x)$ and $f_{0} \in N(K)^{\perp}$. Theorem 1.4 implies $f_{0}$ can be approximated as closely as desired by (finite) linear combinations of the $k_{x}$ 's.

Example 1.5. To illustrate Theorem 1.4 consider the rank-2 integral operator $(K f)(x)=\int_{0}^{1}(x-y) f(y) d y$ mapping $L_{2}(0,1)$ into $L_{2}(0,1)$, Lebesgue measure. It is easy to verify that $K$ satisfies property (C), so the hypotheses of Theorem 1.4 are satisfied. Here, $k_{x}(y)=x-y$, and it is easily shown that

$$
\text { closed } \operatorname{span}\left\{k_{x}: 0 \leq x \leq 1\right\}=\operatorname{span}\{1, y\}=N(K)^{\perp} .
$$

LEMMA 1.6. Let $g, h_{1}, h_{2}, \ldots, h_{n}$ be elements in a Hilbert space $H$ with the inner product of $u, v \in H$ denoted by $(u, v)$. Then the orthogonal projection of $g$ onto $\operatorname{span}\left\{h_{1}, \ldots, h_{n}\right\}$ is given by $\sum_{j=1}^{n} a_{j} h_{j}$, where vector $\left(a_{1}, \ldots, a_{n}\right)^{T}$ is a solution of the linear system $A x=b$ with $a_{i j}=\left(h_{j}, h_{i}\right)$ and $b_{i}=\left(g, h_{i}\right)$.

Proof. Let $P g=\sum_{j=1}^{n} a_{j} h_{j}$ be the orthogonal projection of $g$ onto $\operatorname{span}\left\{h_{1}, \ldots, h_{n}\right\}$. Then for each $i, g-P g$ is perpendicular to $h_{i}$, that is,

$$
0=\left(g-P g, h_{i}\right)=\left(g, h_{i}\right)-\left(P g, h_{i}\right)=\left(g, h_{i}\right)-\sum_{j=1}^{n}\left(h_{j}, h_{i}\right) a_{j} .
$$

From this the lemma follows. 
Observe that the $n \times n$ matrix $A$ in Lemma 1.6 is a Grammian matrix and hence is always nonnegative semidefinite and is positive definite if and only if $\left\{h_{1}, \ldots, h_{n}\right\}$ is a linearly independent set.

The next theorem shows how to choose constants $c_{1}, \ldots, c_{n}$ so that $\sum_{j=1}^{n} c_{j} k_{x_{j}}$ will best approximate the minimum norm solution $f_{0}$ of $K f=g$ in the $L_{2}$-norm without knowing $f_{0}$, that is, only $g(x)$ and the kernel $k(x, y)$ need to be known to determine the $c_{j}$ 's. Most expansion methods for solving first-kind integral equations pick the constants $c_{1}, \ldots, c_{n}$ to minimize the norm of the residual instead of the norm difference from the desired solution. Let $\mathbf{c}=\left(c_{1}, c_{2}, \ldots, c_{n}\right)^{T}$, $\mathbf{b}=\left(g\left(x_{1}\right), g\left(x_{2}\right), \ldots, g\left(x_{n}\right)\right)^{T}$, and let $A$ be the $n \times n$ matrix whose $(i, j)$ th component is given by $\left(k_{x_{j}}, k_{x_{i}}\right)$. With this notation the following theorem can be easily stated.

THEOREM 1.7. If $f_{0}$ is the minimum norm solution of $(K f)(x)=g(x)$, then the minimum over all constants $c_{1}, \ldots, c_{n}$ of $\left\|f_{0}-\sum_{j=1}^{n} c_{j} k_{x_{j}}\right\|$ occurs when $\mathbf{c}$ is a solution of $A \mathbf{x}=\mathbf{b}$. Moreover, the value of $f_{n}=\sum_{j=1}^{n} c_{j} k_{x_{j}}$ is independent of which solution $\mathbf{c}$ of $A \mathbf{x}=\mathbf{b}$ one takes.

Proof. By elementary properties of Hilbert spaces, $\left\|f_{0}-\sum_{j=1}^{n} c_{j} k_{x_{j}}\right\|$ is minimized when the $c_{j}$ 's are chosen so that $\sum_{j=1}^{n} c_{j} k_{x_{j}}$ is the orthogonal projection of $f_{0}$ onto $\operatorname{span}\left\{k_{x_{1}}, \ldots, k_{x_{n}}\right\}$. By Lemma 1.6, $\mathbf{c}=\left(c_{1}, \ldots, c_{n}\right)^{T}$ is a solution of $A \mathbf{x}=\mathbf{b}$, where $b_{i}=\left(f_{0}, k_{x_{i}}\right)$. But

$$
\left(f_{0}, k_{x_{i}}\right)=\int_{Y} f_{0}(y) k_{x_{i}}(y) d \mu(y)=\int_{Y} k\left(x_{i}, y\right) f_{0}(y) d \mu(y)=g\left(x_{i}\right),
$$

and the proof of the first sentence in Theorem 1.7 is complete.

Let $f_{n}^{1}=\sum_{j=1}^{n} c_{j} k_{x_{j}}$ and $f_{n}^{2}=\sum_{j=1}^{n} d_{j} k_{x_{j}}$, where $\mathbf{c}$ and $\mathbf{d}$ are solutions of $A \mathbf{x}=\mathbf{b}$. Then for each $i=1,2, \ldots, n$

$$
\begin{aligned}
\left(f_{n}^{1}-f_{n}^{2}, k_{x_{i}}\right) & =\sum_{j=1}^{n} c_{j}\left(k_{x_{j}}, k_{x_{i}}\right)-\sum_{j=1}^{n} d_{j}\left(k_{x_{j}}, k_{x_{i}}\right) \\
& =g\left(x_{i}\right)-g\left(x_{i}\right)=0 .
\end{aligned}
$$

Since $f_{n}^{1}$ and $f_{n}^{2}$ are in the span of $k_{x_{1}}, \ldots, k_{x_{n}}$, it follows that $f_{n}^{1}=f_{n}^{2}$ and the proof of Theorem 1.7 is complete.

The above argument does not obviously generalize to finding the least squares solution of minimum norm (see [4]) of $K f=g$. The reason for this is that if $f_{0}$ is the least squares solution of minimum norm of $K f=g$, then $\left(K f_{0}\right)(x)=(P g)(x)$ where $P g$ is the orthogonal projection of $g$ onto the closure of the range of the operator $K$. Applying the same argument as in the proof of Theorem 1.7 yields that the $c_{j}$ 's are a solution of $A \mathbf{x}=\mathbf{b}$ where $A$ is as defined immediately preceding Theorem 1.7 , but $b_{i}$ now is $(P g)\left(x_{i}\right)$, which is unknown.

Notice that in Theorem 1.7, the vector formed from the $c_{j}$ 's can be any solution of $A \mathbf{x}=\mathbf{b}$. If the $k_{x j}$ 's are linearly dependent, then $A$ will be a singular matrix. From an application point of view, it is desirable for the $k_{x_{j}}$ 's to be linearly independent so that $A$ will be positive definite and $A \mathbf{x}=\mathbf{b}$ can be solved numerically using the Cholesky decomposition. 
Example 1.8. If $x_{1}, \ldots, x_{n}$ are distinct numbers in the interval $[a, b]$, then $\left\{e^{x_{1} y}, \ldots, e^{x_{n} y}\right\},\left\{\cos \left(x_{1} y\right), \ldots, \cos \left(x_{n} y\right)\right\}$, and $\left\{\sin \left(x_{1} y\right), \ldots, \sin \left(x_{n} y\right)\right\}, x_{j} \neq 0$, are linearly independent sets in $L_{2}(a, b)$, Lebesgue measure.

Suppose $c_{1} e^{x_{1} y}+\cdots+c_{n} e^{x_{n} y}=0$ for all $y \in[a, b]$. Differentiate this equation $i$ times with respect to $y, i=1,2, \ldots, n-1$, to obtain a system of $n$ linear equations which can be written in the form $A \mathbf{x}=0$, where $A$ is a Vandermonde matrix and the $i$ th component of $\mathbf{x}$ is $c_{i} e^{x_{i} y}$. It follows that each $c_{i}=0$. The remaining two sets of functions are shown to be linearly independent in a similar fashion except the original equation should be differentiated $4,8,12, \ldots, 4(n-1)$ times instead of $1,2,3, \ldots,(n-1)$ times with respect to $y$.

A question of practical importance is: For a given value of $n$, how should $x_{1}, \ldots, x_{n}$ be chosen so that $\sum_{j=1}^{n} c_{j} k_{x_{j}}$ can best approximate the minimum norm solution, $f_{0}$, of the integral equation $K f=g$ ? Consider the case $n=1$. Applying Theorem 1.7 to the case $n=1$, one obtains that

$$
f_{1}(y)=\frac{g\left(x_{1}\right)}{\left(k_{x_{1}}, k_{x_{1}}\right)} k_{x_{1}}(y)
$$

is the best $L_{1}$-norm approximation to $f_{0}(y)$ for a given value $x_{1}$, assuming $k_{x_{1}} \neq 0$, hence $\left(k_{x_{1}}, k_{x_{1}}\right)=\left\|k_{x_{1}}\right\|^{2}>0$. Therefore, we want to determine the value of $x_{1}$ which will minimize

$$
\left\|f_{0}-\frac{g\left(x_{1}\right)}{\left(k_{x_{1}}, k_{x_{1}}\right)} k_{x_{1}}\right\| .
$$

Using the fact that $\left(f_{0}, k_{x_{1}}\right)=g\left(x_{1}\right)$, one obtains

$$
\left\|f_{0}-\frac{g\left(x_{1}\right)}{\left(k_{x_{1}}, k_{x_{1}}\right)} k_{x_{1}}\right\|^{2}=\left\|f_{0}\right\|^{2}-\frac{2 g\left(x_{1}\right)^{2}}{\left\|k_{x_{1}}\right\|^{2}}+\frac{g\left(x_{1}\right)^{2}}{\left\|k_{x_{1}}\right\|^{2}}=\left\|f_{0}\right\|^{2}-\frac{g\left(x_{1}\right)^{2}}{\left\|k_{x_{1}}\right\|^{2}} .
$$

Notice that $\left\|f_{0}\right\|^{2}$ being unknown does not hamper solving this minimization problem; find the value of $x_{1}$ that maximizes $g\left(x_{1}\right)^{2} /\left\|k_{x_{1}}\right\|^{2}$. Example 1.5 is simple enough that one can calculate this value of $x_{1}$ exactly. For example, the minimum norm solution of

$$
\int_{0}^{1}(x-y) f(y) d y=5 x-3, \quad 0 \leq x \leq 1,
$$

if $f_{0}(y)=2+6 y$. Using differential calculus, one can show that the maximum of $g(x)^{2} /\left\|k_{x}\right\|^{2}, 0 \leq x \leq 1$, occurs when $x=0$. Thus, the best approximation of $f_{0}(y)$ by functions of the form $\left(g(x) /\left\|k_{x}\right\|^{2}\right) k_{x}(y)$ is $\left(g(0) /\left\|k_{0}\right\|^{2}\right) k_{0}(y)=9 y$. Then $\left\|f_{0}\right\|=\sqrt{28}$ and $\left\|f_{0}-f_{1}\right\|=1$.

Consider $f_{n}=\sum_{j=1}^{n} c_{j} k_{x_{j}}$ for $n>0$, c a solution of $A \mathbf{x}=\mathbf{b}$ and $f_{0}$ the minimum norm solution of $K f=g$. Since $f_{n}$ is the orthogonal projection of $f_{0}$ onto $M_{n} \equiv \operatorname{span}\left\{k_{x_{1}}, \ldots, k_{x_{n}}\right\},\left(f_{0}-f_{n}, f_{n}\right)=0$ and $\left(f_{n}, f_{0}\right)=\left\|f_{n}\right\|^{2}$. Then,

$$
\begin{aligned}
\left\|f_{0}-f_{n}\right\|^{2} & =\left(f_{0}-f_{n}, f_{0}\right)-\left(f_{0}-f_{n}, f_{n}\right) \\
& =\left(f_{0}-f_{n}, f_{0}\right)=\left\|f_{0}\right\|^{2}-\left(f_{n}, f_{0}\right) \\
& =\left\|f_{0}\right\|^{2}-\sum_{j=1}^{n} c_{j}\left(k_{x_{j}}, f_{0}\right)=\left\|f_{0}\right\|^{2}-\sum_{j=1}^{n} c_{j} g\left(x_{j}\right) .
\end{aligned}
$$


This proves the following:

THEOREM 1.9. To minimize $\left\|f_{0}-\sum_{j=1}^{n} c_{j} k_{x_{j}}\right\|$ over all $c_{1}, \ldots, c_{n}$ and $x_{1}, \ldots$, $x_{n}$, one need only solve: maximize $\sum_{j=1}^{n} c_{j} g\left(x_{j}\right)$ subject to $A \mathbf{c}=\mathbf{b}$ over all $x_{1}, \ldots, x_{n}$.

2. Numerical Results. All computer runs were done in double precision on a National Advanced Systems AS/9160 using the VS-FORTRAN version 1.41 compiler (with optimization level $=0$ ) and using the MVS/XA operating system. Double-precision arithmetic on this machine means about 15 decimal digits of accuracy. The parameter $N$ is the number of basis functions, $k_{x_{j}}$ 's, that were used. The $N \times N$ linear system $A \mathbf{x}=\mathrm{b}$, see Theorem 1.7 for notation, can be singular. By Theorem 1.7, it does not matter which solution of $A \mathbf{x}=\mathbf{b}$ is used. Since $A$ can be singular, $A \mathbf{x}=\mathbf{b}$ was numerically solved by computing the singular value decomposition of $A$ (with the LINPACK routine DSVDC) and then computing the minimun norm solution. Owing to the finite precision of the computer's arithmetic, some computed singular values may be small instead of zero. Computed singular values that gave the smallest residual, $\|\mathbf{b}-A \mathbf{x}\|$, were used. E04JAF from the Numerical Algorithms Group (NAG) Library was used to maximize $\sum_{j=1}^{n} c_{j} g\left(x_{j}\right)$ subject to $A \mathbf{c}=\mathbf{b}$. All integral equations tested were of the form $\int_{0}^{1} k(x, y) f(y) d y=g(x)$, $0 \leq x \leq 1$. Therefore,

$$
a_{i j}=\left(k_{x_{j}}, k_{x_{i}}\right)=\int_{0}^{1} k\left(x_{j}, y\right) k\left(x_{i}, y\right) d y .
$$

For all numerical examples tested, this integral was evaluated exactly and no quadrature method was needed. For the purpose of evaluating the algorithm, the minimum norm solution, $f_{0}(y)$, was entered into the program as a function statement and $b_{i}=g\left(x_{i}\right)$ was calculated by evaluating

$$
g\left(x_{i}\right)=\int_{0}^{1} k\left(x_{i}, y\right) f(y) d y
$$

exactly, where $f(y)$ is some given function used to determine $g(x)$ and may be different from $f_{0}(y)$.

Once the $c_{1}, \ldots, c_{n}$ had been calculated by solving $A \mathbf{x}=\mathbf{b}$, the error between the minimum norm solution $f_{0}(y)$ and the approximate solution,

$$
f_{n}(y)=\sum_{j=1}^{n} c_{j} k_{x_{j}}(y)
$$

was approximated by calculating $f_{0}(y)-f_{n}(y)$ for $y \in S$ where $S=\{0.00,0.05,0.10$, $0.15,0.20, \ldots, 1.00\}$. (Using an increment of .01 instead of .05 had little effect on the results.)

The following table lists all of the integral equations tested, along with how well the method worked. EX denotes the example number. $f_{0}$ denotes the minimum norm solution; $k(x, y)$ denotes the kernel function. $\mathrm{AE}=$ $\max \left\{\left|f_{0}(y)-f_{n}(y)\right|: y \in S\right\}$, an approximation to the maximum absolute error. $\mathrm{RE}=\max \left\{\left|f_{0}(y)-f_{n}(y)\right| /\left|f_{0}(y)\right|: y \in S, \quad f_{0}(y) \neq 0\right\}$, an approximation to the relative error. Both $\mathrm{AE}$ and $\mathrm{RE}$ are rounded to one decimal place. RES denotes the approximation to the residual given by $\max \left\{\left|\left(K f_{n}\right)(x)-g(x)\right|: x \in S\right\}$. $\mathrm{N}$ is the 
number of basis functions that yielded the best number for both $\mathrm{AE}$ and RE (there was no ambiguity for these test problems). A listing of the FORTRAN source code is available upon request.

\begin{tabular}{rccccccr}
\hline EX & $f(y)$ & $f_{0}(y)$ & $k(x, y)$ & $\mathrm{AE}$ & $\mathrm{RE}$ & $\mathrm{RES}$ & $\mathrm{N}$ \\
\hline 1 & $2+6 y$ & $f(y)$ & $x-y$ & $3 \times 10^{-15}$ & $1 \times 10^{-15}$ & $1 \times 10^{-15}$ & 2 \\
2 & $e^{y}$ & $(18-6 e) y$ & $x-y$ & $2 \times 10^{-15}$ & $1 \times 10^{-15}$ & $3 \times 10^{-16}$ & 2 \\
& & $+(4 e+10)$ & & & & & \\
3 & $2+3 y$ & $f(y)$ & $(x-y)^{3}$ & $3 \times 10^{-13}$ & $1 \times 10^{-13}$ & $2 \times 10^{-14}$ & 5 \\
4 & 1 & $f(y)$ & $\cos (x y)$ & $3 \times 10^{-12}$ & $3 \times 10^{-12}$ & $1 \times 10^{-15}$ & 3 \\
5 & $y$ & $f(y)$ & $\sin (x y)$ & $8 \times 10^{-9}$ & $1 \times 10^{-8}$ & $4 \times 10^{-15}$ & 8 \\
6 & $(\cos y) / 3$ & $f(y)$ & $\cos (x y)$ & $1 \times 10^{-10}$ & $7 \times 10^{-10}$ & $3 \times 10^{-16}$ & 4 \\
7 & $\cos y-.5 \cos \left(\frac{y}{2}\right)$ & $f(y)$ & $\cos (x y)$ & $3 \times 10^{-10}$ & $3 \times 10^{-9}$ & $8 \times 10^{-16}$ & 4 \\
8 & $e^{y}$ & $f(y)$ & $e^{x y}$ & $1 \times 10^{-9}$ & $5 \times 10^{-10}$ & $9 \times 10^{-16}$ & 5 \\
9 & $y^{2}+2 y-1$ & $f(y)$ & $(x-y)^{2}$ & $4 \times 10^{-15}$ & $6 \times 10^{-14}$ & $2 \times 10^{-16}$ & 4 \\
10 & $\sin y$ & $f(y)$ & $e^{x y}$ & $2 \times 10^{-6}$ & $4 \times 10^{-5}$ & $8 \times 10^{-13}$ & 9 \\
11 & $\cos y$ & $f(y)$ & $e^{x y}$ & $7 \times 10^{-6}$ & $7 \times 10^{-6}$ & $1 \times 10^{-13}$ & 15 \\
12 & $y^{2}+1$ & $f(y)$ & $\cos (x y)$ & $2 \times 10^{-8}$ & $1 \times 10^{-7}$ & $6 \times 10^{-13}$ & 5 \\
13 & $\cos (2 y)$ & $f(y)$ & $\cos (x y)$ & $6 \times 10^{-5}$ & $4 \times 10^{-4}$ & $2 \times 10^{-13}$ & 6 \\
14 & $1-2 y$ & $f(y)$ & $e^{x y}$ & $5 \times 10^{-6}$ & $3 \times 10^{-5}$ & $2 \times 10^{-12}$ & 10 \\
15 & $e^{2 y}$ & $f(y)$ & $e^{x y}$ & $4 \times 10^{-5}$ & $3 \times 10^{-5}$ & $2 \times 10^{-12}$ & 10 \\
16 & $\sin (2 y)$ & $f(y)$ & $\sin (x y)$ & $7 \times 10^{-6}$ & $1 \times 10^{-5}$ & $2 \times 10^{-13}$ & 8 \\
\hline
\end{tabular}

To give an illustration of some of the details involved, consider Example 4. (All numbers have been rounded.) For $N=1$, the best $x_{0}$ was 0.0068 . For $N=$ 2 , the best $\left(x_{0}, x_{1}\right)$ was $(0,0000,0.9392)$. For $N=3$, the best $\left(x_{0}, x_{1}, x_{2}\right)$ was $(0.0000,0.6530,0.5127)$. The various errors and residuals were

\begin{tabular}{r|ccc}
\hline & $N=1$ & $N=2$ & $N=3$ \\
\hline $\mathrm{AE}$ & $1.5 \times 10^{-5}$ & $4.4 \times 10^{-12}$ & $2.7 \times 10^{-12}$ \\
$\mathrm{RE}$ & $1.5 \times 10^{-5}$ & $4.4 \times 10^{-12}$ & $2.7 \times 10^{-12}$ \\
$\mathrm{RES}$ & $9.6 \times 10^{-7}$ & $1.2 \times 10^{-15}$ & $1.0 \times 10^{-15}$ \\
\hline
\end{tabular}

3. Conclusions. An advantage of the above method is that it automatically provides a set of basis functions that span the orthogonal complement of the null space of the integral operator $K$. The test examples listed above confirm that this method will work well if the minimum norm solution can be well approximated by a few of the basis functions, $k_{x}, x \in X$; otherwise, solutions with high precision cannot be found with this method. Since the $N \times N$ matrix $A$ used to numerically calculate the $c_{j}$ 's is a Grammian matrix (with respect to a finite-dimensional weighted inner product space), $A$ becomes numerically ill-conditioned when $N$ is very large. Experiments with some test problems indicate that $N \geq 25$ is normally too large. A second advantage of this method is that the coefficients of the $k_{x_{j}}$ 's were calculated to minimize the norm difference of the approximate solution to the minimum norm solution, instead of choosing them to minimize the residual. The third advantage of this method is that it provides a way of picking "optimal" basis functions for a fixed value of $N$. 
When performing these tests, the minimum norm solution, $f_{0}$, was used to determine the value of $N$ which yielded the best approximation, $f_{1}$, to $f_{0}$. This was done to obtain an accurate evaluation of the method. In applications, $f_{0}$ will be unknown, but a value of $N$ can be determined by choosing it to minimize the residual $\left\|K f_{1}-g\right\|$, or at least an approximation to it. This was done (as described in Section 2) for all examples tested.

Acknowledgments. I would like to thank my graduate student Jae-Heon Yun for making these computer runs and for observing that $f_{n}$ is independent of which solution of $A \mathbf{x}=\mathbf{b}$ is used; see Theorem 1.7.

Department of Mathematics and Computation Center

Iowa State University

Ames, Iowa 50011

E-mail: gmgrl@isumvs.bitnet

1. R. S. ANDERssen \& P. M. PREnTER, "A formal comparison of methods proposed for the numerical solution of first kind integral equations," J. Austral. Math. Soc. Ser. B, v. 22, 1981, pp. $488-500$.

2. C. W. GROETSCH, "Uniform convergence of regularization methods for Fredholm equations of the first kind," J. Austral. Math. Soc. Ser. A, v. 39, 1985, pp. 282-286.

3. GLENN R. LUECKE \& KEVIN R. HICKEY, "A note on the filtered least squares minimal norm solution of first kind equations," J. Math. Anal. Appl., v. 100, no. 2, 1984, pp. 635-641.

4. GLENN R. LUECKE \& KEVIN R. HICKEY, "Convergence of approximate solutions of operator equations," Houston J. Math., v. 11, no. 3, 1985, pp. 345-353.

5. MANFRED R. TRUMMER, "A method for solving ill-posed linear operator equations," SIAM J. Numer. Anal., v. 21, no. 4, 1984, pp. 729-737. 